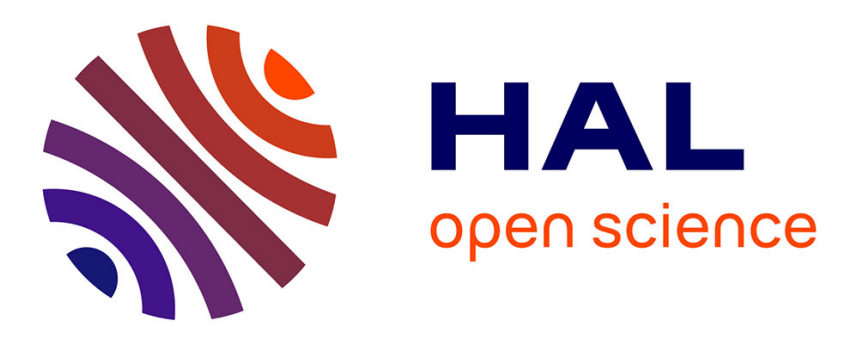

\title{
Détermination du frottement intérieur des systèmes à fort amortissement; cas des mélanges à base d'eau
}

\author{
J.L. Bouvier-Volaille, R. Point, R. Vassoille
}

\section{To cite this version:}

J.L. Bouvier-Volaille, R. Point, R. Vassoille. Détermination du frottement intérieur des systèmes à fort amortissement; cas des mélanges à base d'eau. Revue de Physique Appliquée, 1985, 20 (8), pp.537-544. 10.1051/rphysap:01985002008053700 . jpa-00245368

\section{HAL Id: jpa-00245368 https://hal.science/jpa-00245368}

Submitted on 1 Jan 1985

HAL is a multi-disciplinary open access archive for the deposit and dissemination of scientific research documents, whether they are published or not. The documents may come from teaching and research institutions in France or abroad, or from public or private research centers.
L'archive ouverte pluridisciplinaire HAL, est destinée au dépôt et à la diffusion de documents scientifiques de niveau recherche, publiés ou non, émanant des établissements d'enseignement et de recherche français ou étrangers, des laboratoires publics ou privés. 


\title{
REVUE DE PHYSIQUE APPLIQUÉE
}

\section{Détermination du frottement intérieur des systèmes à fort amortissement ; cas des mélanges à base d'eau}

\author{
J. L. Bouvier-Volaille $\left({ }^{*}\right)$, R. Point $\left(^{+}\right)$et R. Vassoille $\left({ }^{*}\right)$ \\ $\left(^{*}\right)$ Groupe d'Etude de Métallurgie Physique et de Physique des Matériaux (**), \\ $\left({ }^{+}\right)$Laboratoire d'Informatique Appliquée, \\ I.N.S.A., Bât. 502, 69621 Villeurbanne Cedex, France
}

(Reçu le 27 septembre 1984, révisé le 8 février 1985, accepté le 7 mai 1985)

\begin{abstract}
Résumé. - Les mesures de frottement intérieur donnent beaucoup d'informations concernant les propriétés des solides en relation avec leur microstructure. Dans certaines conditions la valeur de l'amortissement peut être très élevée. C'est en particulier le cas de systèmes à base d'eau (glace au voisinage du point de fusion, mélanges utilisés en cryobiologie...). Le but de l'étude suivante est de présenter un appareillage capable de mesurer le frottement intérieur de tels matériaux dans une large gamme de valeurs et de proposer ensuite quelques exemples d'application.

Abstract. - Internal friction measurements give many informations concerning the properties of solids in relation to their microstructure. Under some conditions, the value of damping may be very important. In this paper, a torsional pendulum apparatus able to measure internal friction in a large range of values is described. Some applications to the study of ice near the melting point and water based composite materials (such as water mixtures used in cryobiology) are presented.
\end{abstract}

\section{Introduction.}

L'étude du frottement intérieur des solides est une méthode qui depuis plus d'un demi-siècle a fourni de nombreuses données sur les propriétés des solides en relation avec leur microstructure [1-3]. Diverses techniques de mesure ont été mises en œuvre et suivant le domaine de fréquence on s'intéresse soit à l'atténuation et à la vitesse de propagation des ondes ultrasonores dans le matériau (Megahertz), soit aux vibrations de barreaux (de plus de $100 \mathrm{kHz}$ à $10^{-5} \mathrm{~Hz}$ ). L'étude des propriétés micromécaniques de la glace $I_{h}$ a été abordée au Laboratoire à l'aide de la technique $\mathrm{du}$ frottement intérieur dans le domaine des ultrasons [4], dans le domaine des très basses fréquences $[5,6]$ et à une fréquence voisine de $1 \mathrm{~Hz}[7,8]$. Ce matériau présente souvent au voisinage du point de fusion un amortissement élevé. Par exemple, dans

(**) Unité associée au C.N.R.S. no 341. le cas des monocristaux déformés plastiquement, le mouvement à courte distance des dislocations entraîne une dissipation d'énergie qui peut être très importante [9]. D'autre part, dans le cas des polycristaux, nous avons mis en évidence l'existence d'un pic lié à la présence des joints de grains [10]. Dans ce cas aussi, l'amortissement interne peut être très important.

Un autre type d'étude impliquant des matériaux présentant un amortissement élevé a aussi été réalisé : il s'agit du comportement anélastique d'échantillons préparés à partir de solutions de composés pouvant être employés en cryobiologie comme cryoprotecteurs (éthylène-glycol, glycérol, DMSO). En effet, il est possible d'étudier à l'aide de mesures de frottement intérieur les conditions d'obtention et de stabilité de l'état vitreux formé après trempe dans des îlots dispersés au sein d'une matrice de glace [11-13]. Là encore, on est en présence d'amortissements très élevés, en particulier dans la zone de transition vitreuse. Les mesures correspondant aux études pré- 
cédentes ont été réalisées à partir des enregistrements de la décroissance de l'amplitude des oscillations du pendule. Dans ces conditions, le calcul du frottement intérieur n'était pas automatique et la précision restait médiocre.

La nécessité de disposer d'un appareillage permettant de mesurer avec une égale précision et de manière automatique des valeurs du frottement intérieur pouvant varier de $10^{-3}$ à des valeurs supérieures à 0,5 (en décrément logarithmique) s'est donc imposée. Nous allons donc décrire le dispositif que nous avons réalisé au laboratoire et présenter une extension de l'étude précédemment réalisée sur les mélanges eauéthylène-glycol [11].

\section{Dispositif expérimental.}

Le dispositif expérimental comporte essentiellement deux parties. La première concerne l'ensemble pendulaire auquel se rattache la régulation de température et la seconde se rapporte à l'équipement électronique d'excitation et d'entretien des oscillations ainsi que la mesure du frottement intérieur et la période des oscillations.

2.1 DesCRIPTION DU SYSTÈME PENDULAIRE. - Les mesures de frottement interne sont effectuées à l'aide d'un pendule de torsion inversé fonctionnant à une fréquence voisine de $1 \mathrm{~Hz}$. L'échantillon étudié sert d'élément de rappel de l'ensemble pendulaire. Cet échantillon (lame de glace ou lame de composite glace-matière vitreuse) se présente sous la forme d'un parallèlépipède de $2 \times 8 \times 76 \mathrm{~mm}^{3}$ et est obtenu soit par taille mécanique dans des blocs de glace soit par moulage pour les mélanges eau-alcools étudiés [7, 11]. L'échantillon est placé dans un four dont on peut réguler la température (palier, montée ou descente régulée). $\mathrm{La}$ régulation de température se fait par chauffage à partir de la température de l'azote liquide. La vitesse de variation de la température peut varier de $0,16 \mathrm{~K} / \mathrm{min}$ jusqu'à $10 \mathrm{~K} / \mathrm{min}$.

\subsection{DISPOSITIF D'ENTRETIEN D'ASSERVISSEMENT ET DE MESURE}

2.2.1 Description générale. - Les mesures de frottement intérieur sont faites en décroissance libre, mais il faut préalablement entretenir les oscillations de l'ensemble pendulaire à amplitude constante. Le mouvement oscillatoire du pendule est détecté par des cellules différentielles (Fig. 1) dont la position centrale est contrôlée par un suiveur de zéro, B. Le signal sinusoïdal amplifié provenant des cellules A est utilisé pour asservir le pendule à amplitude constante (la déformation $\varepsilon$ de l'échantillon est d'environ $2 \times 10^{-5}$ ). Les mesures de frottement intérieur sont faites à des temps fixes programmés par l'ordinateur. Un ensemble d'interfaces permet l'acquisition de la période du pendule, de la température de l'échantillon et des

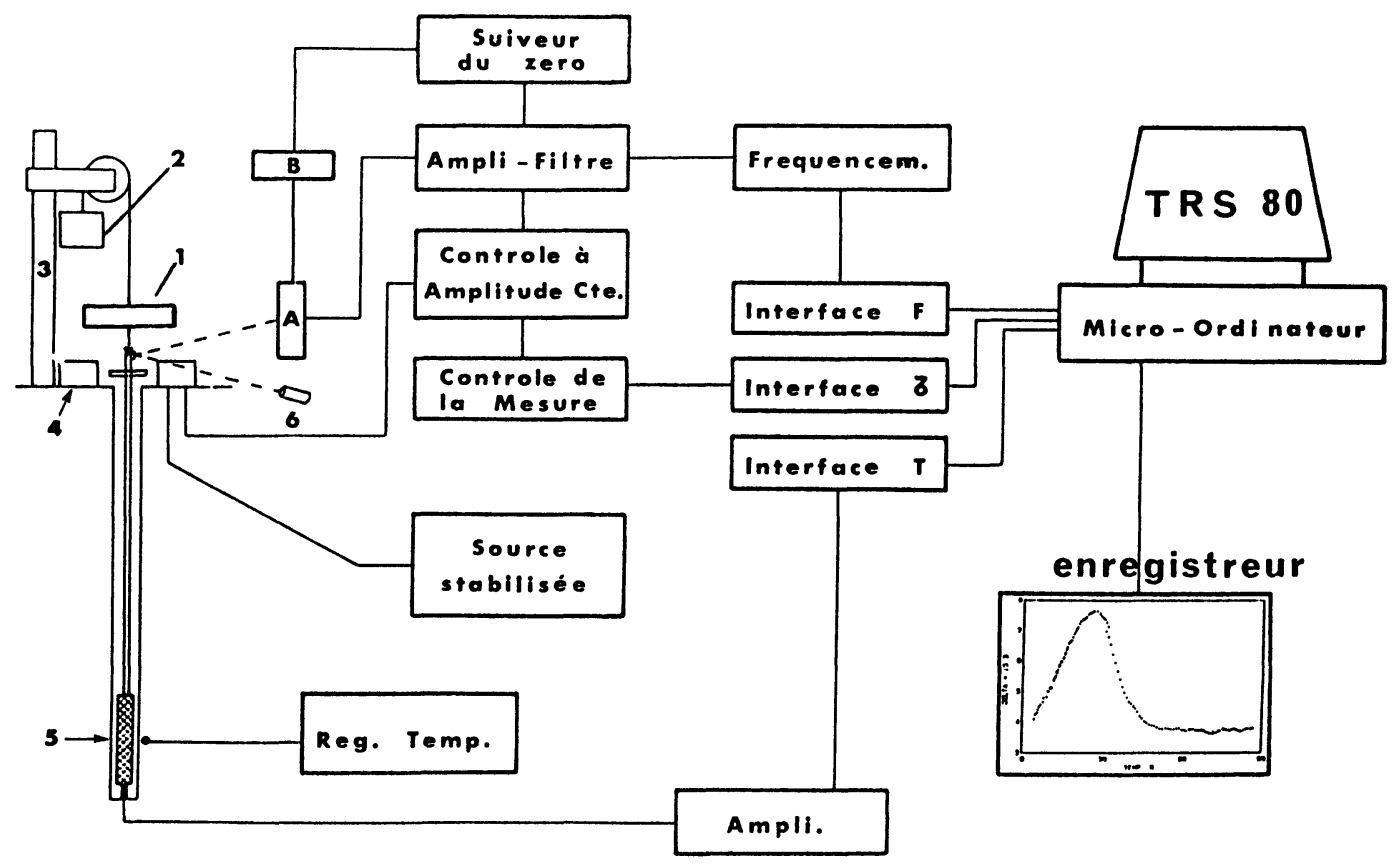

Fig. 1. - Schéma général de l'installation $: 1:$ masse d'inertie ; 2 : contre-poids ; $3:$ support ; 4 : bobines d'excitation du mouvement pendulaire agissant sur des aimants permanents; $5:$ four pour la régulation de température; $6:$ projecteur envoyant un faisceau lumineux permettant la détection du mouvement à l'aide des cellules différentielles $\mathrm{A} ; \mathrm{B}:$ niveau de zéro.

[Schematic representation of internal friction apparatus $: 1:$ inertia mass $; 2:$ counterpoise ; $3:$ support ; $4:$ magnetic coils, oscillations are produced by means of magnet sets in the magnetic field of the coils; $5:$ temperature regulation; $6:$ searchlight ; A : differential photovoltaic detector ; B : detection of the position specimen.] 
amplitudes crêtes positives et négatives lors des décroissances libres. Ces données sont traitées par un micro-ordinateur TRS 80 et tracées sur un enregistreur pour un contrôle immédiat.

2.2.2 Description des interfaces. - Les interfaces ont été réalisées à partir des signaux présents sur le connecteur arrière du TRS 80. Outre les adresses et données amplifiées, on dispose de deux signaux $\mathrm{IN}^{*}$ et OUT* déjà décodés qui simplifient à la fois l'interfaçage matériel et le logiciel par l'existence des instructions IN et OUT en BASIC. La figure 2 donne le schéma général de l'interfaçage du TRS 80 . On dispose en sortie de deux signaux analogiques permettant la

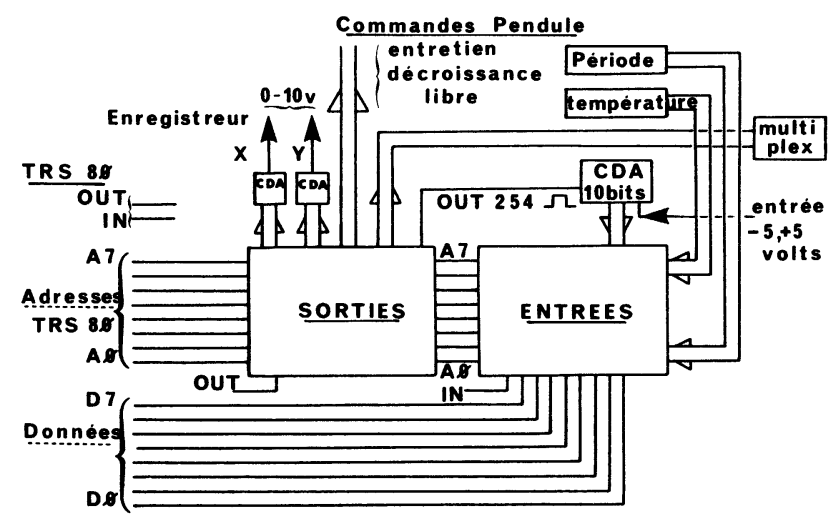

Fig. 2. - Schéma général de l'interfaçage du TRS 80.

[Schematic diagram of the connection of the TRS 80.] commande d'un enregistreur $X Y$, et de signaux logiques permettant le contrôle du pendule, de l'ensemble de multiplexage de la température, de la période et du déclenchement du convertisseur analogique digital 10 bits. En entrées, on trouve les signaux logiques provenant du multiplexeur température/ période et du convertisseur analogique digital. L'acquisition de la température et de la période est faite sous contrôle du BASIC à l'aide des instructions OUT pour l'envoi des adresses et IN pour la lecture des données (Fig. 3).

Pour éviter des erreurs de mesure, on bloque le transfert des données de température pendant leur adressage, tandis que l'on teste la fin de mesure de période avant son acquisition.

La mesure des amplitudes crêtes positives et négatives est faite entièrement par logiciel. Souvent les mesures de frottement intérieur sont faites avec une précision de l'ordre du $1 \%$ ce qui justifie l'utilisation d'un convertisseur 10 bits. Pour la mesure de l'amplitude crête, 50 acquisitions par demi-période suffisent pour obtenir 2 ou 3 mesures de la valeur crête avec une précision de $1 \%$.

La demande de conversion est totalement asynchrone vis-à-vis du pendule et se fait avec une vitesse telle que l'on obtient plus de 200 acquisitions par période. On teste chaque nouvelle acquisition par rapport à la précédente en fonction du signe de l'amplitude $(+$ ou -$)$ : on détecte ainsi les valeurs crêtes positives et négatives qui sont alors rangées en mémoire pour un traitement ultérieur en BASIC. Afin

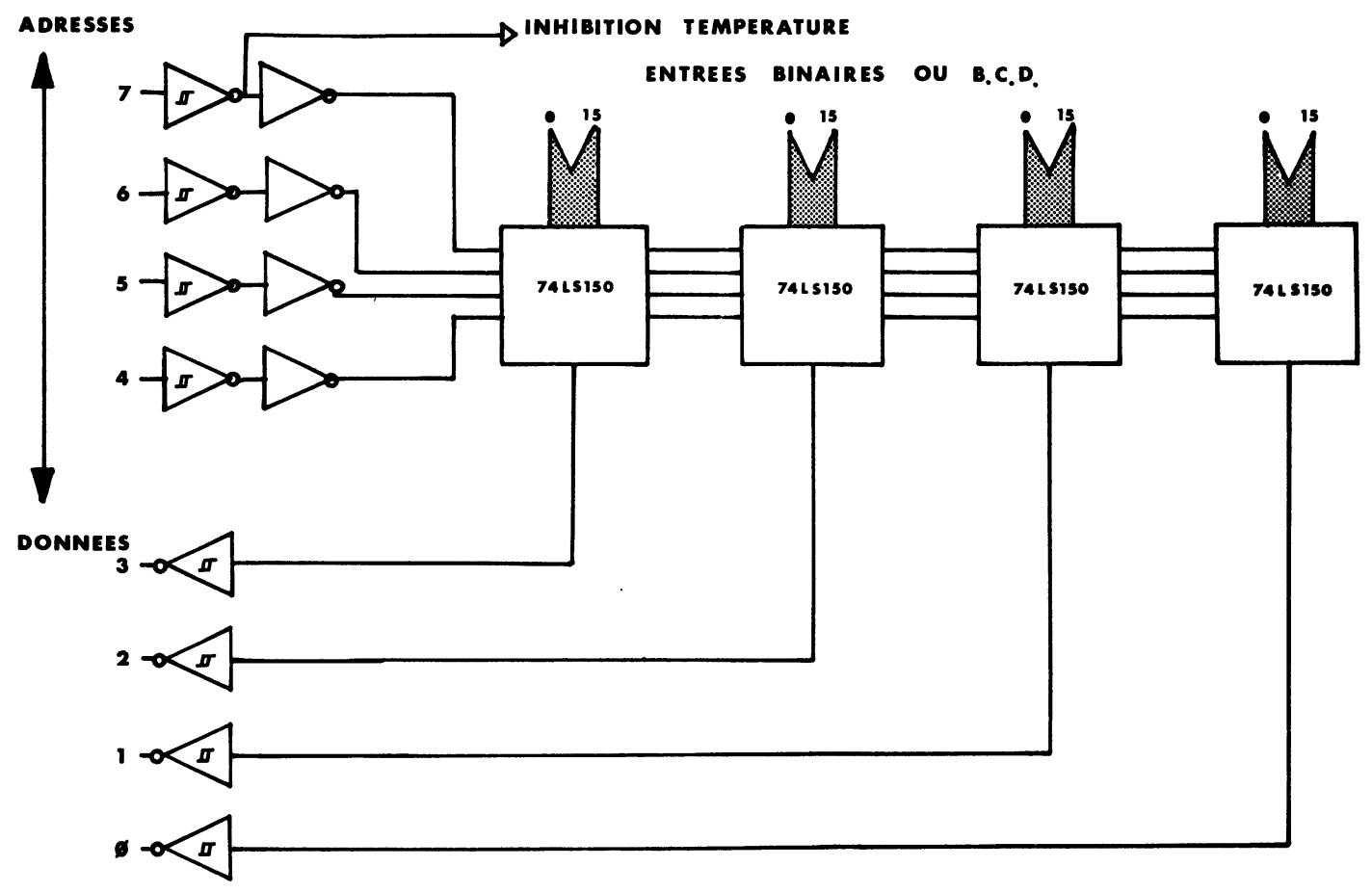

Fig. 3. - Carte du multiplexage période-température.

[Schematic diagram of the multiplexage period-temperature.] 
d'éviter des temps de mesure trop longs, on arrête l'acquisition après 60 oscillations ou, par exemple, lorsque l'amplitude crête devient inférieure à $80 \%$ de l'amplitude crête du début de décroissance libre.

Nous verrons plus loin que lorsque le frottement intérieur devient très important on opère un changement de méthode de mesure. On fait alors un échantillonnage de la décroissance libre. Les valeurs acquises sur la sinusoïde amortie en décroissance libre sont rangées en mémoire pour un traitement par BASIC. Le temps séparant la prise des échantillons, et le nombre d'échantillons, sont entièrement programmables par l'utilisateur.

Nous allons maintenant présenter les méthodes de mesure du décrément logarithmique employé.

\section{Mesure du décrément logarithmique $\delta$ des oscilla- tions.}

Lorsqu'on travaille en régime d'oscillations libres, on utilise couramment le décrément logarithmique des oscillations amorties pour caractériser le frottement intérieur. En effet, $\delta=1 / 2 \Delta W / W$ où $\Delta W$ est l'énergie dissipée au cours d'un cycle et $W$ est l'énergie élastique maximale mise en jeu au cours de ce cycle. Par ailleurs $\delta=\operatorname{Ln}\left(A_{n} / A_{n+1}\right)$ où $A_{n}$ et $A_{n+1}$ sont respectivement les amplitudes des $n$-ième et $(n+1)$-ième oscillations de l'ensemble pendulaire.

Pour évaluer $\delta$ on peut employer diverses méthodes et pour augmenter la précision on a intérêt à utiliser un grand nombre d'oscillations. Cela suppose (i) que $\delta$
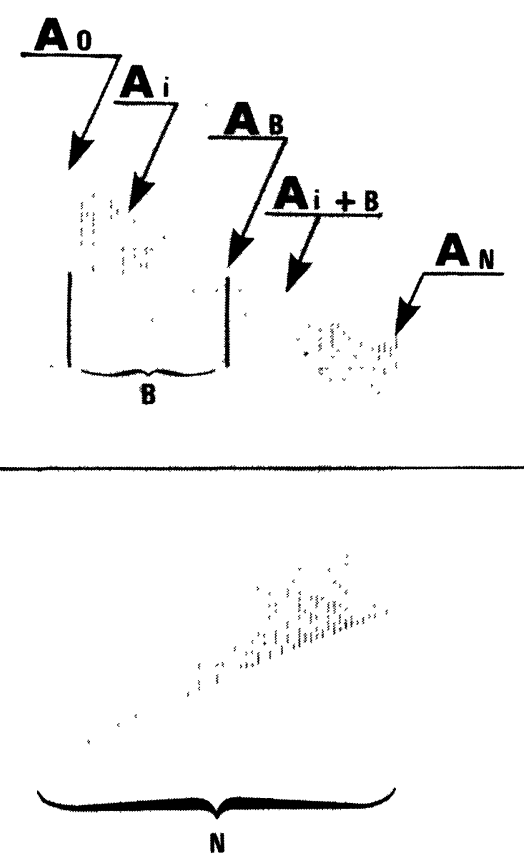

Fig. 4. - Décroissance de l'amplitude $A_{i}$ des oscillations libres de l'ensemble pendulaire.

[Decrease of the amplitude $A_{i}$ of the free oscillations of the pendulum.] est indépendant de l'amplitude $\varepsilon$ de déformation. Pour ce qui concerne la glace, nous avons montré [9] qu'à $1 \mathrm{~Hz}$ la dépendance de $\delta$ avec l'amplitude $\varepsilon$ ne devient notable que lorsque $\varepsilon$ dépasse $5 \times 10^{-5}$ et ceci même pour des températures élevées. Ainsi pour nos mesures faites avec $\varepsilon_{\max }=2 \times 10^{-5}$ on pourra considérer cette condition réalisée; (ii) que $\delta$ reste suffisamment faible pour que le nombre d'oscillations pouvant être valablement utilisé soit assez grand. Or, dans le cas des échantillons de glace polycristalline par exemple, l'amortissement lié à la présence des joints de grains conduit à des valeurs de $\delta$ qui peuvent être supérieures à 0,5 [10]. C'est aussi ce qui se produit pour des échantillons réalisés à partir des mélanges eau-alcools [14]. Il est alors nécessaire de mettre en œuvre un calcul différent. Nous allons présenter successivement les méthodes que nous avons choisies.

3.1 Calcul de $\delta$ dans le Cas des amortissements FAIBLES. - Dans le cas des faibles amortissements, le décrément logarithmique $\delta$ des oscillations s'évalue en appliquant la formule classique :

$$
\delta=\operatorname{Ln}\left(A_{n} / A_{n+1}\right) .
$$

Dans le but de minimiser les erreurs liées à de petites instabilités mécaniques, on peut répéter ce calcul sur un ensemble de $N$ oscillations (Fig. 4); alors :

$$
\begin{aligned}
\delta & =\operatorname{Ln}\left(A_{0} / A_{1}\right)=\cdots=\operatorname{Ln}\left(A_{B-1} / A_{B}\right)= \\
& =1 / B \operatorname{Ln}\left(A_{0} / A_{B}\right)=1 / B \operatorname{Ln}\left(A_{i} / A_{B+i}\right)
\end{aligned}
$$

d'où l'expression finale

$$
\delta=1 / B^{2} \sum_{i=0}^{B-1} \operatorname{Ln}\left(A_{i} / A_{B+i}\right) .
$$

On obtient ainsi une moyenne de la valeur de $\delta$. Les valeurs de $N$ et $B$ peuvent être ajustées en fonction de l'amortissement considéré. Un test d'amplitude permet, si l'amortissement est trop élevé (ce qui signifie que le nombre d'oscillations est trop faible), de procéder au deuxième type de calcul que nous allons préciser.

3.2 AMORTISSEMENT TRÈS ÉLEVÉ. - Lorsque le système étudié est fortement amorti, la méthode précédente devient inexploitable. Il faut alors mettre en ouvre des programmes plus sophistiqués, utilisant par exemple la FFT, pour aboutir, par analyse de la décroissance libre, à un calcul précis du décrément. De tels programmes, assez volumineux, sont difficiles à implanter sur un petit système, et leur temps d'exécution en BASIC interprété risque d'être assez long. Cela pénalise grandement leur application pour un traitement automatique en temps réel.

Aussi, nous avons mis au point un programme simple, peu volumineux, écrit en BASIC, et dont le temps d'exécution est acceptable. Un calcul du décré- 
ment logarithmique, effectué sur 250 points expérimentaux, dure $1 \mathrm{~min} 30 \mathrm{~s}$ environ. Ce programme peut être implanté sur n'importe quel micro-ordinateur.

La courbe expérimentale obtenue point par point est de la forme :

$$
y_{i}=y_{0} \mathrm{e}^{-\tau t i} \cos \omega t_{i} .
$$

Une division par $\cos \omega t_{i}$ ne conserve que la composante exponentielle de la courbe ; on obtient les points :

$$
y_{i}^{*}=y_{0} \mathrm{e}^{-\tau t_{i}} .
$$

(La division par $\cos \omega t_{i}$ réclame que ce terme soit non nul : du fait de la représentation interne des nombres en machine, limitée à un certain nombre de bits, les valeurs trop faibles de $\cos \omega t_{i}$ conduisent à des points aberrants qu'il faut éliminer.)

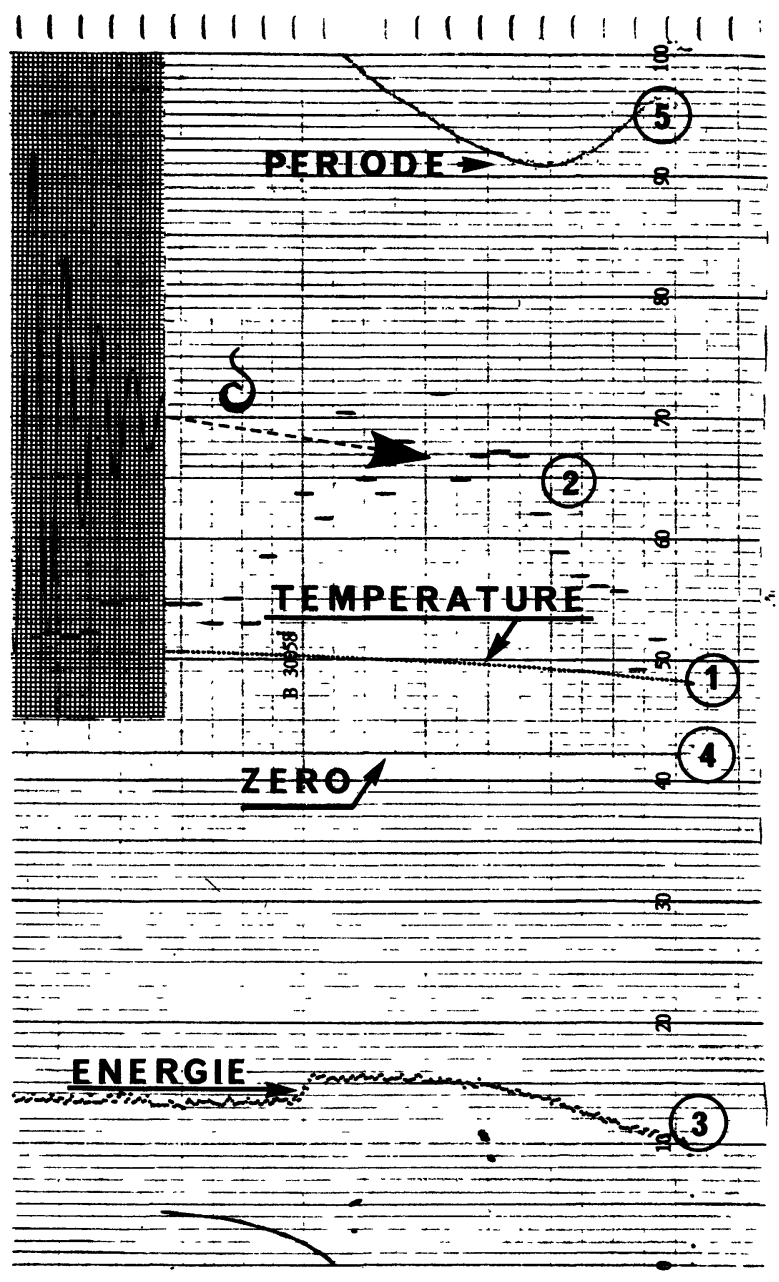

a)

Fig. 5. - Exemples d'enregistrements du frottement intérieur et de la période en fonction de la température : a) pic de frottement intérieur de niveau élevé $\left(\delta_{\max }\right.$ env. 0,68$)$; b) frottement intérieur de niveau moyen : étude de la dispersion à température constante.
On prend ensuite le logarithme népérien des valeurs $y_{i}^{*}$ retenues, soit :

$$
Y_{i}=\operatorname{Ln} y_{i}^{*}=\operatorname{Ln}\left(y_{0} \mathrm{e}^{-\tau t_{i}}\right)
$$

donc

$$
Y_{i}=-\tau t_{i}+\operatorname{Ln} y_{0} \text { où } \operatorname{Ln} y_{0}=\text { Cte. }
$$

Les points $Y_{i}$ se distribuent selon une droite dont on désire connaître le coefficient directeur $\tau$.

Un programme classique de régression linéaire, appliqué aux couples de valeurs $\left(Y_{i}, t_{i}\right)$, permet de déterminer $\tau$ d'où la valeur de décrément logarithmique.

3. 3 RÉSULTAT : DISPERSION DES MESURES. - Au cours d'une manipulation, cinq données sont enregistrées ainsi que le montre la figure $5 \mathrm{a}: 1)$ la température, 2) le décrément logarithmique $\delta, 3$ ) l'énergie fournie

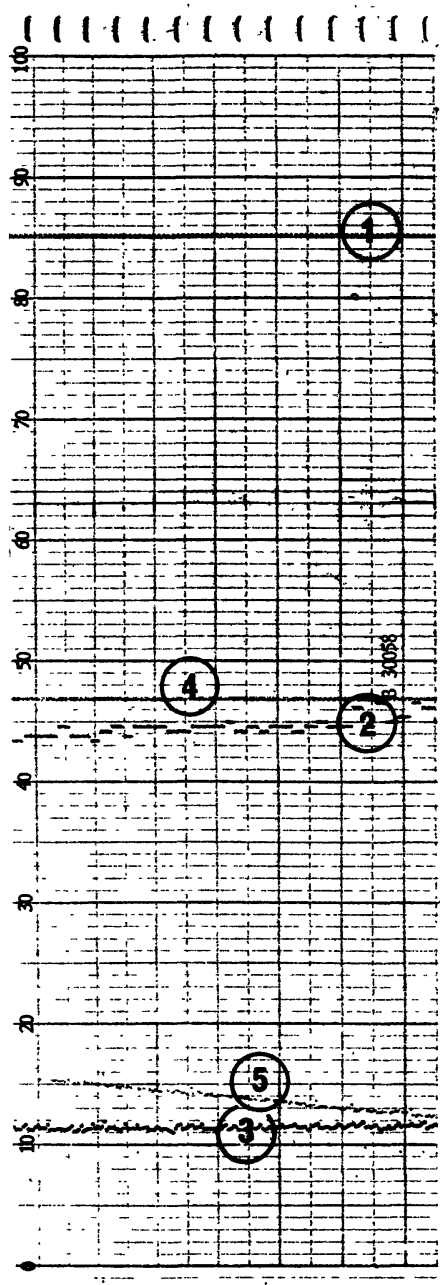

b)

[Examples of internal friction and period recording versus temperature : a) high damping corresponding to internal friction peak $\left(\delta_{\max }\right.$ env. 0.68$)$; b) medium damping : study of the dispersion of internal friction measurements at constant temperature.] 
aux bobines d'excitation du mouvement pendulaire, 4) la position de l'éprouvette et 5) la période des oscillations. Pour l'échantillon servant de test dans le cas de la figure $5 \mathrm{a}$, on observe un pic de frottement intérieur avec un amortissement maximal moyen $\delta_{\max }=0,68$. A partir de cet exemple, nous estimons la valeur moyenne de la dispersion des valeurs de $\delta$ : elle est de l'ordre de $5 \times 10^{-3}$ ce qui est satisfaisant compte tenu de la valeur élevée du frottement intérieur. Cette dispersion moyenne des valeurs mesurées de $\delta$ est bien plus faible lorsque l'amortissement est moins élevé comme le montre la figure $5 \mathrm{~b}$ ou $\delta=0,25$. Dans ce cas en effet, cette dispersion des mesures est inférieure à $10^{-3}$.

\section{Exemples d'applications aux mélanges à base d'eau.}

Afin de donner quelques exemples d'application de l'appareillage nous allons présenter trois résultats qui illustrent bien les possibilités de mesure de frottement intérieur dans une large gamme de valeurs de l'amortissement.

4.1 Pic de joints de grains dans le cas des polyCRISTAUX DE GLACE $\mathrm{I}_{\mathrm{h}}$. - Dans l'étude du comportement anélastique des polycristaux de glace $I_{h}$ rappelée en introduction [10] nous avons montré que l'amortissement dépendait, entre autres facteurs, de la surface de joints de grains. Cependant, nous avons montré que l'intensité de relaxation peut diminuer considérablement lorsque la densité de dislocations est importante (joints de grains réalisés par soudure de deux monocristaux), ou lorsque l'on est en présence de noeuds triples. Ceci est confirmé par la figure 6 repré-

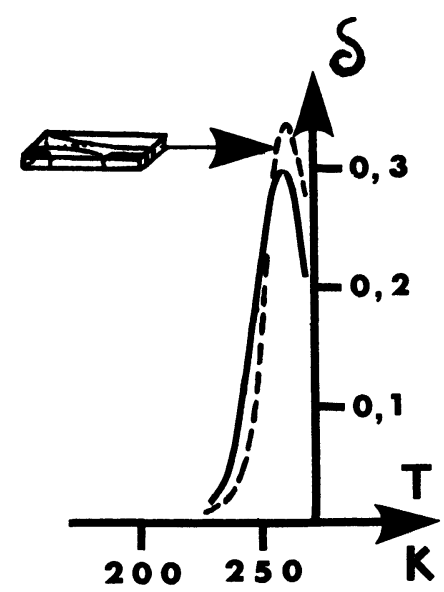

Fig. 6. - Frottement intérieur haute température dans le cas de la glace. La courbe en trait plein correspond au pic de joints de grains dans le ças du givre compacté. La courbe en traits pointillés correspond au cas d'un polycristal à gros grains.

[High temperature internal friction spectrum of the polycrystalline ice : grain boundaries peak : - full line : hoarfrost; - dashed line : specimen containing only some big grains.] sentant le spectre de frottement intérieur d'un échantillon réalisé avec du givre compacté (dimension des grains de l'ordre de quelques dizaines de $\mu \mathrm{m}$ ). Dans ce cas, l'amortissement maximal est voisin de 0,3 , ce qui est sensiblement plus faible que ce que l'on mesure dans le cas d'échantillons ne comportant que quelques joints de grains [10].

4.2 Cas du mélange eau-glycérol. - Dans un travail récent [13] nous avons étudié le comportement anélastique d'échantillons réalisés par trempe de solutions de glycérol de concentration variable mais faible (de 0,5 à $3 \%$ en poids de glycérol). Le glycérol est un alcool très employé en cryobiologie; en effet, le taux de survie cellulaire obtenu après maintien à basse température est généralement très élevé lorsque le glycérol est employé comme cryoprotecteur [15].

Le spectre de frottement interne obtenu en fonction de la température montre l'existence de trois pics (Fig. 7). Le premier, situé vers $160 \mathrm{~K}$ est un pic de relaxation correspondant aux mouvements de rotation des molécules d'eau sous l'action de la contrainte de mesure [7]. Le second, situé vers $189 \mathrm{~K}$ est associé à la transition vitreuse des îlots du mélange eauglycérol dispersés dans la matrice de glace constituant l'essentiel de l'échantillon. L'amplitude de ce pic dépend de la concentration du mélange initial comme le montre la figure 7 mais aussi du traitement thermique subi par l'échantillon [13]. Enfin, le troisième

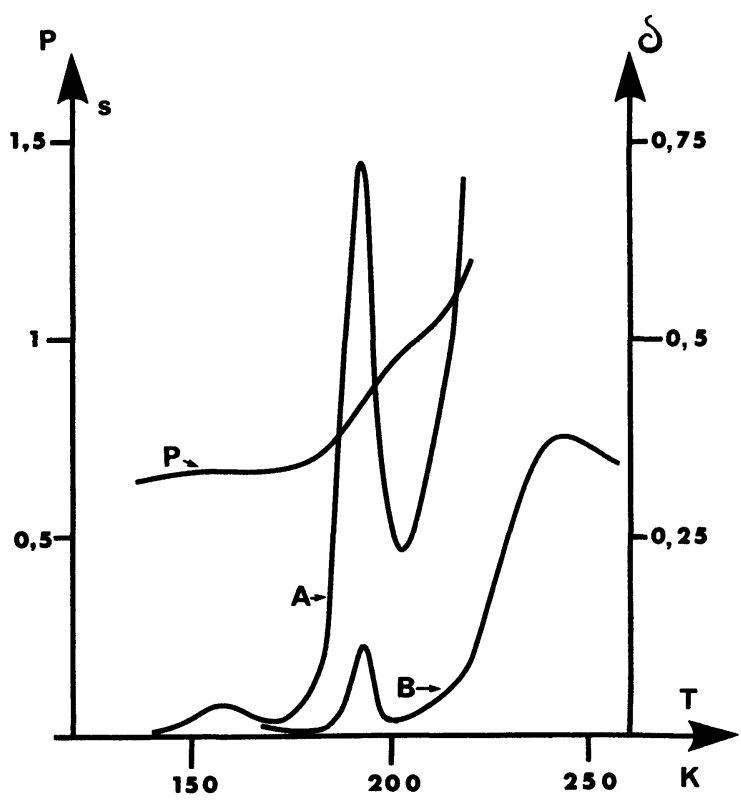

Fig. 7. - Spectre de frottement intérieur du mélange eauglycérol en fonction de la température. A : solution initiale contenant $3 \%(\mathrm{~W} / \mathrm{W})$ de glycérol. B : solution initiale contenant $0,5 \%(\mathrm{~W} / \mathrm{W})$ de glycérol. $P$ est la période des oscillations du pendule.

[Water-glycerol mixture internal friction spectrum versus temperature; the glycerol concentration of the solution is : $\mathrm{A}: 3 \%(\mathrm{~W} / \mathrm{W}) ; \mathrm{B}: 0.5 \%(\mathrm{~W} / \mathrm{W}) . P$ is the period of pendulum oscillations.] 
pic est dû à la présence des joints de grains dans l'échantillon.

Nous constatons donc sur la figure 7 que le frottement interne varie considérablement dans toute l'étendue du domaine de températures considérées, ce qui justifie l'intérêt des deux dispositifs de calcul précédemment détaillés.

\subsection{ANALYSE DU COMPORTEMENT DU MÉLANGE EAU-} ÉTHYLÈNE-GLYCOL APRÈS TRAITEMENT THERMIQUE. Dans une étude antérieure le comportement anélastique d'échantillons réalisés à partir de solutions faiblement concentrées d'éthylène-glycol [11] avait été analysé en référence avec celui de la glace pure [7]. D'importantes modifications du spectre de frottement intérieur ont été observées : en particulier, vers $170 \mathrm{~K}$ il apparaît un pic $P_{G}$ dont l'amplitude croît avec la concentration en éthylène-glycol. Ce pic a été associé à la transition vitreuse du mélange eau-éthylène-glycol. A plus haute température, la valeur du frottement intérieur augmente de manière considérable. La dissipation d'énergie correspondante est pour l'essentiel due à la présence des joints de grains plus ou moins saturés en éthylène-glycol. A cause de l'imprécision des mesures effectuées au cours du travail précité, nous n'avions pas analysé le spectre de frottement intérieur de tels mélanges après traitements thermiques. Ceux-ci ont été pratiqués en vue de préciser les conditions de dévitrification de la partie

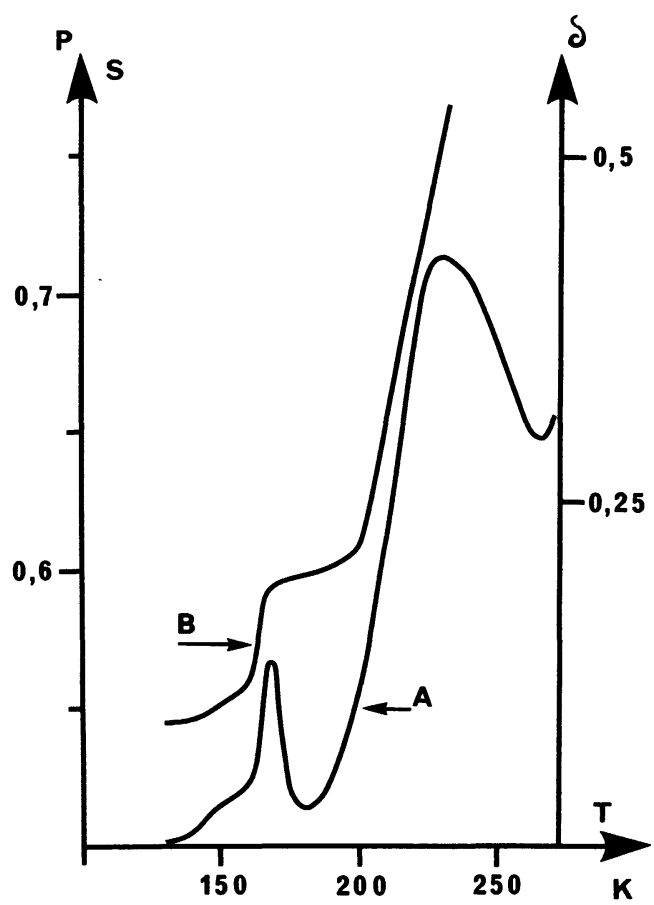

Fig. 8. - Comportement anélastique d'un échantillon fabriqué par trempe d'une solution d'éthylène-glycol à $0,5 \%(W / W)$. A : Frottement intérieur; B : Période des oscillations du pendule.

[Inelastic behaviour of water-ethylene-glycol mixture $(0.5 \% \mathrm{~W} / \mathrm{W})$ versus temperature. A : internal friction; B : period of oscillations.] vitreuse des échantillons. La figure 8 montre le spectre tracé pour une lame obtenue par trempe d'une solution contenant $0,5 \%$ en poids d'éthylène-glycol. A partir des données tirées de l'étude des conditions de stabilité de l'état vitreux [12] nous avons maintenu l'échantillon $4 \mathrm{~h}$ à $163 \mathrm{~K}$ (température pour laquelle la vitesse de germination est maximale) et $17 \mathrm{~h}$ à $181 \mathrm{~K}$ (où la vitesse de croissance est maximale). Dans ces conditions on obtient le spectre représenté sur la figure 9. On constate les modifications suivantes :

a) Le pic $P_{G}$ a disparu comme nous l'avions antérieurement observé [11]; ceci avait été interprété en terme de cristallisation des îlots vitreux dispersés dans l'échantillon; cette cristallisation est confirmée par la diminution de la valeur de la période des oscillations du pendule (augmentation du module de l'échantillon).

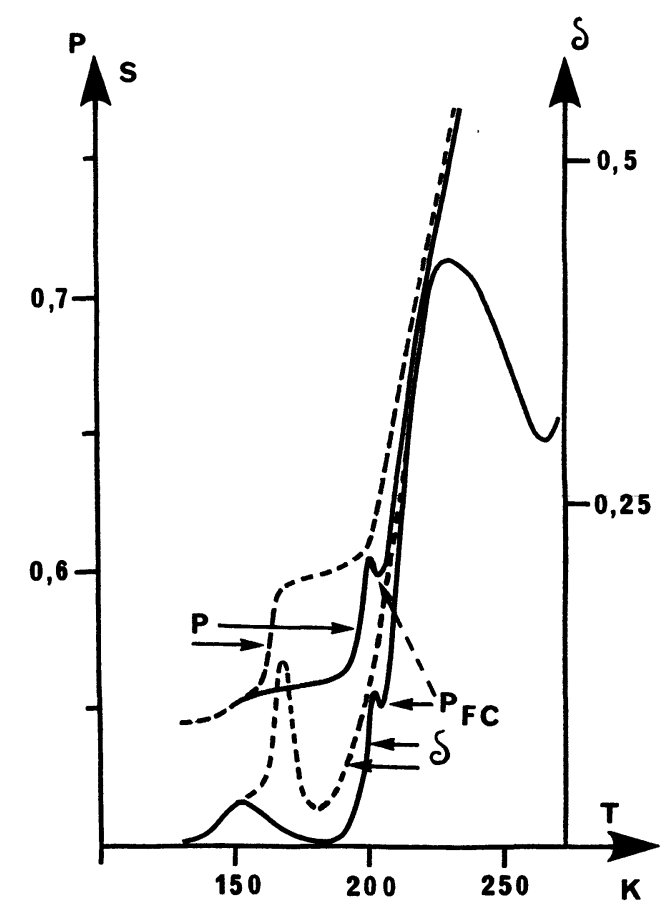

Fig. 9. - Evolution du spectre de frottement intérieur et de la période des oscillations en fonction de la température après traitement thermique : courbes en traits pointillés : état initial (Fig. 8); courbes en trait plein : après $4 \mathrm{~h}$ à $163 \mathrm{~K}$ et $17 \mathrm{~h}$ à $181 \mathrm{~K}$.

[Evolution of the internal friction spectrum after annealing treatment : dashed line : initial state (Fig. 8); full line : after $4 \mathrm{~h}$ at $163 \mathrm{~K}$ and $17 \mathrm{~h}$ at $181 \mathrm{~K}$.]

b) Il apparaît entre 200 et $210 \mathrm{~K}$ un pic de frottement intérieur associé à une anomalie de période. Nous désignerons ce pic par $\mathbf{P}_{\mathrm{FC}}$. Dans le but de déterminer son origine nous avons maintenu l'échantillon à une température voisine de $200 \mathrm{~K}$. On constate sur la figure 10 une diminution du frottement intérieur jusqu'à une valeur "d'équilibre" qui est atteinte en moins d'une heure. Lorsque l'échantillon est maintenu à une température légèrement supérieure 


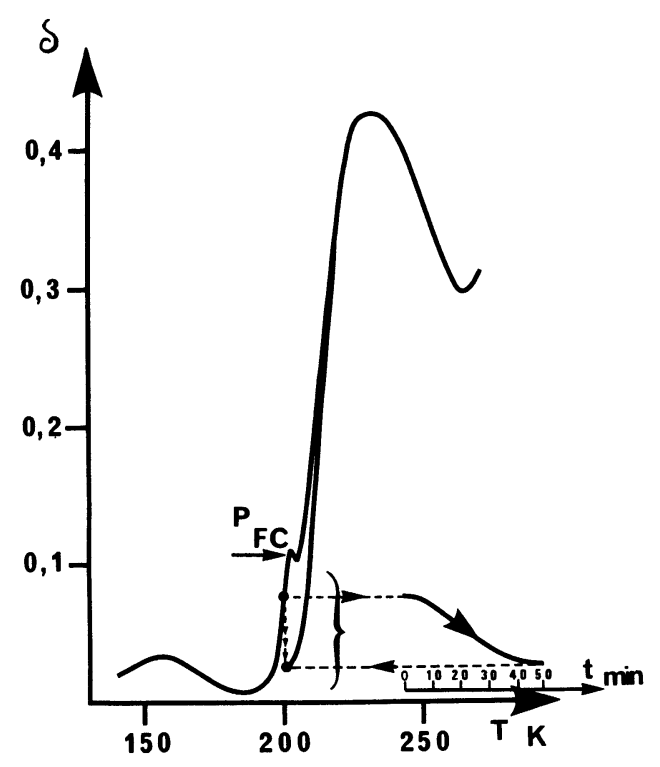

Fig. 10. - Traitement isotherme à $200 \mathrm{~K}$ : évolution du frottement intérieur conduisant à la disparition du pic $P_{F C}$; le graphe situé à droite de la figure représente la cinétique de fusion des îlots cristallisés lors du traitement thermique précédent.

[Annealing treatment at $200 \mathrm{~K}$ : the peak $\mathrm{P}_{\mathrm{FC}}$ disappears; the graphic situated on the right of the figure shows the melting kinetics of crystallized islets during annealing treatment.]

$(205 \mathrm{~K})$ le frottement intérieur diminue aussi mais, comme le montre la figure 11a, la valeur d'équilibre est atteinte plus rapidement. Si l'on refroidit ensuite l'échantillon on constate la réapparition du pic $P_{G}$ tandis que le pic $\mathrm{P}_{\mathrm{FC}}$ tend à disparaître (Fig. 11b). Enfin le pic $P_{G}$ est complètement reconstitué lorsque l'on refroidit l'échantillon à partir d'une température $(212 \mathrm{~K})$ sensiblement supérieure à celle où apparaît $\mathrm{P}_{\mathrm{FC}}$ (Fig. 11c). Nous pouvons donc attribuer le pic $P_{\mathrm{FC}}$ à la fusion de la phase cristallisée du mélange eauéthylène-glycol obtenue à l'issue des maintiens isothermes. Il existe donc un domaine de température

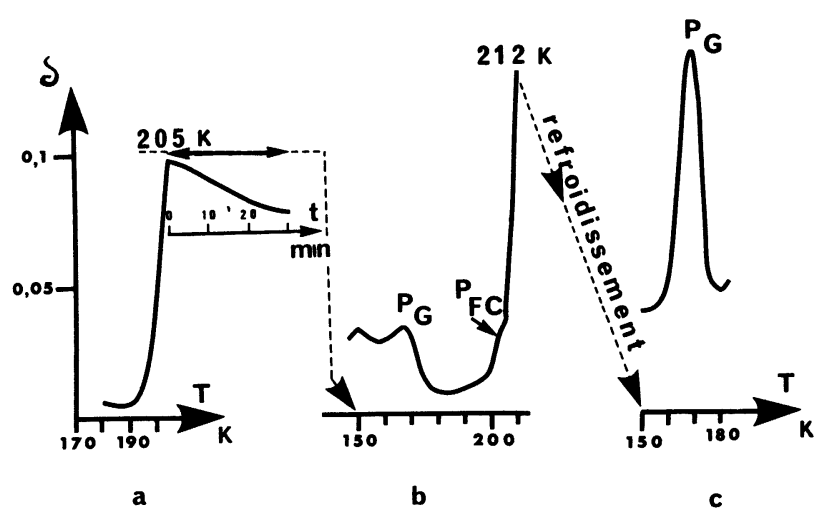

Fig. 11. - Traitements thermiques conduisant à la reconstitution des îlots vitreux : (a) traitement isotherme à $205 \mathrm{~K}$ : fusion partielle des îlots cristallins; (b) après trempe de l'échantillon reconstruction du pic $P_{G}$; (c) reconstruction totale de $P_{G}$ après trempe à partir de $212 \mathrm{~K}$.

[Annealing treatments leading to the formation of vitreous islets from crystallized state :(a) $205 \mathrm{~K}$ annealing : crystallized islets are partially melted; (b) after quenching from $205 \mathrm{~K} \mathrm{P}_{\mathrm{G}}$ is partially restored; (c) after quenching from $212 \mathrm{~K} \mathrm{P}_{\mathrm{G}}$ is completely restored.]

compris entre 200 et $210 \mathrm{~K}$ dans lequel se produit cette fusion : l'anomalie de période liée à $\mathrm{P}_{\mathrm{FC}}$ (Fig. 9) pourrait correspondre à une chute des constantes élastiques due à cette transformation de phase solideliquide.

En conclusion, nous avons donc réalisé un appareillage permettant de mesurer le frottement interne de matériaux susceptibles de présenter, dans le domaine de température exploré, un amortissement très variable, avec la possibilité d'atteindre des valeurs très élevée. C'est le cas des matériaux comme la glace ou les structures composites à base d'eau, pour lesquelles on a montré que le frottement intérieur peut être mesuré dans de bonnes conditions malgré des variations s'étendant sur trois décades depuis $10^{-3}$. Cela nous a permis d'obtenir des informations sur la microstructure ou sur les changements de phase se produisant dans de tels matériaux.

\section{Bibliographie}

[1] Perez, J., Peguin, P., Fantozzi, G. et Gobin, P. F., Ann. Phys. 5 (1970) 303.

[2] Gobin, P. F., Perez, J., Le frottement intérieur. Application à la Métallurgie Physique, Bulletin CEM XII (1972) 1-69.

[3] Fantozzi, G., Esnouf, C., Benoit, W. et Ritchie, G., Prog. Mater. Sci. 27 (1982) 311.

[4] Tatibouet, J., Mai, C., Perez, J. et Vassoille, R., Revue Phys. Appl. 15 (1980) 835.

[5] Tatibouet, J., Perez, J., Vassoille, R., J. Physique Colloq. 42 (1981) C5-541.

[6] Tatibouet, J., Perez, J. et Vassoille, R., J. Physique Colloq. 44 (1983) C9-799.

[7] Vassollle, R., Tatibouet, J., Perez, J. et Gobin, P. F., C.R. Hebd. Séan. Acad. Sci., série B, 278 (1974) 409.

[8] Vassoille, R., Perez, J., Mai, C. et Gobin, P. F ,
Proceedings of Internal friction and ultrasonic attenuation in Solids (University of Tokyo Press) 1977, p. 279.

[9] Vassollle, R., Mai, C. et Perez, J., J. Glaciology 21, $\mathrm{n}^{\circ} 85$ (1978) 375.

[10] Perez, J., Mai, C., Tatibouet, J. et Vassoille, R., Phys. Status Solidi (a) 52 (1979) 321.

[11] Alberola, N., Perez, J., Tatibouet, J. et Vassoille, R., J. Phys. Chem. 86 (1982) 2998.

[12] Alberola, N., Perez, J., Tatibouet, J. et Vassoille, R., J. Phys. Chem. 87 (1983) 4264.

[13] Vassoille, R., Perez, J. et Tatibouet, J., J. Physique Colloq. 44 (1983) C9-341.

[14] Vassoille, R., Alberola, N., Perez, J. et Tatibouet, J., J. Physique Colloq. 44 (1983) C9-341.

[15] MaCGanN, L. E., Cryobiology 15 (1978) 382. 